\title{
State space properties of Flexible Manufacturing Cell based on Hierarchical Timed Colored Petri Nets
}

\author{
Sanjib Kumar Saren ${ }^{1, *}$, Florin Blaga $^{2}$, and Tiberiu Vesselenyi ${ }^{1}$ \\ ${ }^{1}$ Mechatronics Department, University of Oradea, Romania \\ ${ }^{2}$ Industrial Engineering Department, University of Oradea, Romania
}

\begin{abstract}
The subject of this paper is focused on the state space behavioral structure of a flexible manufacturing cell (FMC) using a hierarchical model based on Colored Timed Petri Nets theory. In the paper we discussed various types of properties of the state space in CPNTools and a simulation of the manufacturing cell model with single resource places and transition in order to identify the overall time in the cell. The proposed designed model is based on the manufacturing cell from Faculty of Managerial and Technological Engineering, University of Oradea. In this recent work, we discussed the sequence of resource places and transitions of the flexible manufacturing cell explanation with state space analysis using CPNTools software.
\end{abstract}

\section{Introduction}

The implementation of high level Petri Nets provides ideas and applications in various types of problems to improve Flexible manufacturing system (FMS). The applications of Colored Petri Nets are more and more found in manufacturing sector. State space feature is inbuilt in CPNTools software which provides directed graph with a node with each possible reachable marking in the system. State space tools are used to create reachability, boundedness, home properties, and liveness and fairness properties in the system. The application of state space is explained by Christensen in [1] where the use of state space analysis in Colored Petri Nets is described when the state space is finite and also state space allows the user to analyze the system behavior. In [2] the state space method for Colored Petri Nets using time concept and the condensed infinite to finite state space method is presented. In [3] the authors addressed state space analysis in Colored Petri Nets to define each possible behavioral property for nodes and arcs in the concurrent system. Some major features are described in [4] where Colored Petri Nets integrates items such as liveness properties, meaning absence of the deadlocks, boundedness properties meaning finiteness of buffers and resources required for the model and properness which are explained as the ability to recover from failure.

${ }^{*}$ Corresponding author: sanjibksaren@gmail.com 
In this present work, we briefly discussed the Hierarchy model of a flexible manufacturing cell implementing Colored Timed Petri Nets to observe the detailed behavior of the cell and explain the state space analysis of the model with explanation of various properties related to the flexible cell.

\section{Hierarchical Flexible manufacturing cell designs}

Colored Petri Nets are high level Petri Nets with extension of Place Transitions Petri Nets which allows carrying color token data with values which distinguishes them from other Petri Nets. Temporal behavior of the system can be identified with Colored Petri Nets using delay time and arcs time in the system. Theoretically, the definition of Hierarchical Color Petri Nets (HCPN) is explained elaborately in [5]. A hierarchical CPN has a 9 tuple $=(\mathrm{S}$, SN, SA, PN, PT, PA, FS, FT, and PP) which are explained below:

(a) $S$ is a finite set of pages such that: Each page $s \in S$ is a non-hierarchical $C P N$ :

$\mathrm{CPN}=(\Sigma, \mathrm{P}, \mathrm{T}, \mathrm{A}, \mathrm{N}, \mathrm{C}, \mathrm{G}, \mathrm{E}, \mathrm{I})$ (The non-hierarchical CPN is defined in [5])

The sets of net elements are pair wise disjoints: $\forall \mathrm{s} 1, \mathrm{~s} 2 \in \mathrm{S}:[\mathrm{s} 1 \neq \mathrm{s} 2 \Rightarrow(\mathrm{P} 1 \cup \mathrm{T} 1 \cup \mathrm{A} 1) \cap$ $(\mathrm{P} 2 \cup \mathrm{T} 2 \cup \mathrm{A} 2)=\varnothing]$.

(b) $\mathrm{SN} \subseteq \mathrm{T}$ is a set of substitution nodes.

(c) SA is a page assignment function. It is defined from SN into S such that: No page is a subpage of itself: $\{\mathrm{s} 0, \mathrm{~s} 1 \ldots \mathrm{sn} \in \mathrm{S} * \mid \mathrm{n} \in \mathrm{N}+\wedge \mathrm{s} 0=\mathrm{sn} \wedge \forall \mathrm{k} \in 1 . . \mathrm{n}$ : sk $\in \mathrm{SA}(\mathrm{SNsk}-1)\}=\varnothing$.

(d) $\mathrm{PN} \subseteq \mathrm{P}$ define a set of port nodes.

(e) PT = port type function. It is defined from PN into \{in, out, i/o, general $\}$.

(f) $\mathrm{PA}=$ it's a port assignment function,

(g) FS $\subseteq \mathrm{P}$ is a finite set of fusion sets,

(h) FT = fusion type of function,

(i) $\mathrm{PP} \in \mathrm{SM}$ define multi-set of prime pages.

HCPN are useful to model manufacturing systems with timed constraints and provide high level information about the system. While Coloured Petri Nets used to model the structure and flow in a production system, the Hierarchical Coloured Petri Nets allow the introduction of analysis and information processing. In [6] is described that CPNTools provides to construct hierarchical models. In Figure 1, this manufacturing cell is modeled based on three sub models which are respectively: (a) part arrival (b) part processing and (c) part storage.

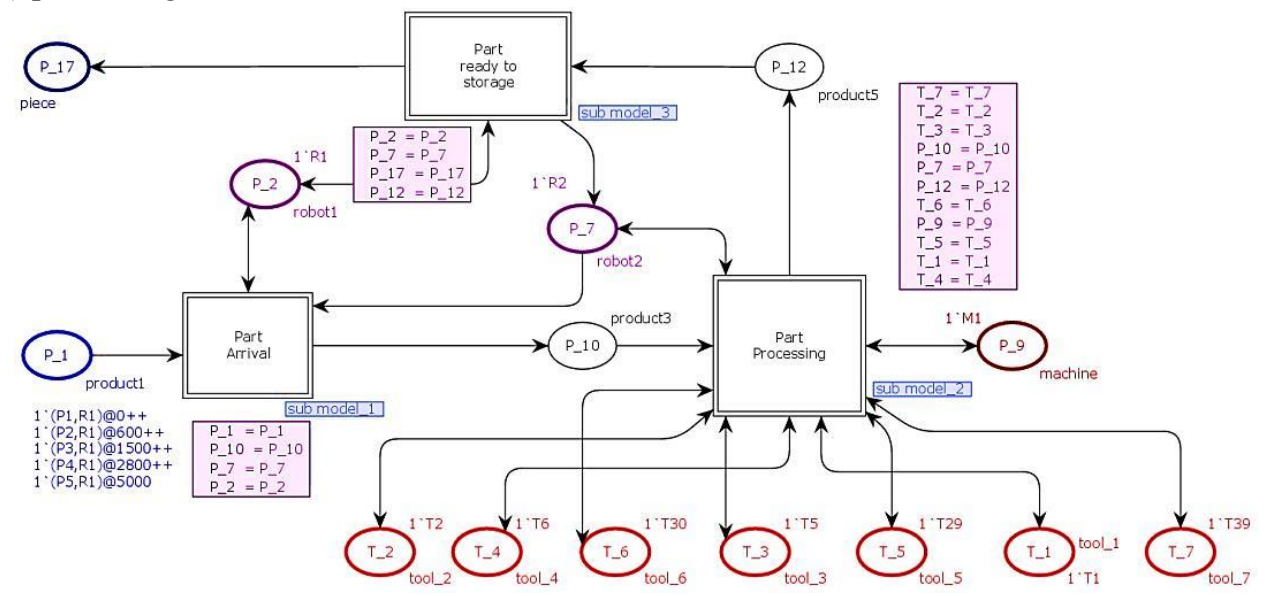

Fig. 1. Hierarchical model of the flexible manufacturing cell. 


\section{Simulation observation of the model}

During the simulation of the hierarchical model of the flexible cell, the number of steps to complete the manufacturing process can be found. During the simulation each sub model according to part arrival in the cell is activated. Each operation is depending on the mentioned color token in resource places and the described time decision in each transition in the cell. The operation steps found in the cell to complete the process is 55 steps. In the followings we provide the information about steps and sequences for the cell. It is clear that in the model we provide the time in transition to execute the operations in the cell and in each case the time involved in transition is observed for all three sub models. The steps provide the behavior of each sub model with respect to color set and variables in the cell. In Figure 2, we represent the observed the time after simulation in the cell and the token value for the respective resources.

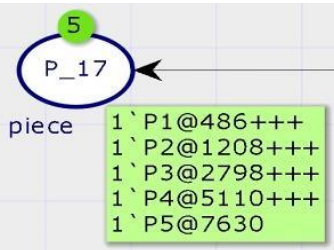

(a)

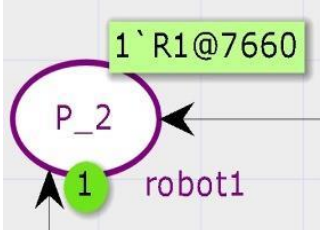

(b)

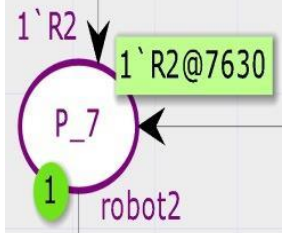

(c)

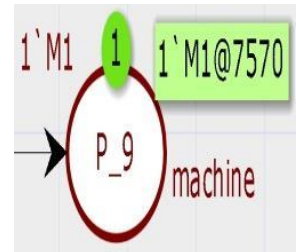

(d)

Fig. 2. Simulation results in (a) Total time in final storage. (b) Time observed for robot1. (c) Time observed for robot2. (d) Time for the machine in hierarchical flexible manufacturing cell model.

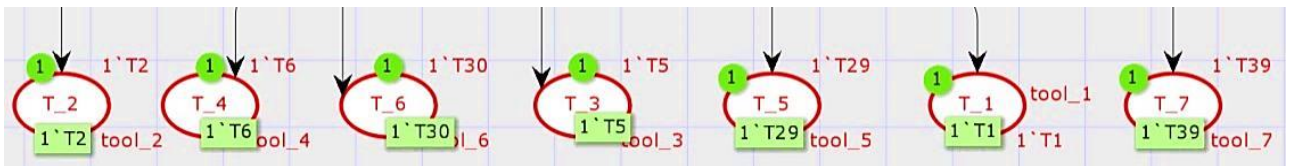

Fig. 3. Tools used for processing of parts containing colour token value after simulation.

The steps and sequences are very important to complete the simulation process for the cell. The model is based on time in each transitions and also resource places. In this hierarchical model during simulation CPNTools provides each sub model operation with the respective to time. The important steps and sequences are mention in Table1.

Table 1. Time and sequences in the cell

\begin{tabular}{|c|c|c|}
\hline Time (Seconds) & Transitions & Sequences \\
\hline 0 & $\mathrm{~T} 1$ & $\left(1: \mathrm{sub} \_\right.$model_1 $)-\mathrm{p}=\mathrm{P} 1-\mathrm{r} 1=\mathrm{R} 1$ \\
\hline 486 & $\mathrm{~T} 11$ & $(1: \mathrm{sub}$ model_3 $-\mathrm{r} 1=\mathrm{R} 1-\mathrm{p}=\mathrm{P} 1$ \\
\hline 1208 & $\mathrm{~T} 11$ & $(1: \mathrm{sub}$ _model_3 $)-\mathrm{r} 1=\mathrm{R} 1-\mathrm{p}=\mathrm{P} 2$ \\
\hline 2798 & $\mathrm{~T} 11$ & $(1: \mathrm{sub}$ _model_3 $)-\mathrm{r} 1=\mathrm{R} 1-\mathrm{p}=\mathrm{P} 3$ \\
\hline 5110 & $\mathrm{~T} 11$ & $(1: \mathrm{sub}$ _model_3 $)-\mathrm{r} 1=\mathrm{R} 1-\mathrm{p}=\mathrm{P} 4$ \\
\hline 7630 & $\mathrm{~T} 11$ & $(1: \mathrm{sub}$ model_3 $)-\mathrm{r} 1=\mathrm{R} 1-\mathrm{p}=\mathrm{P} 5$ \\
\hline
\end{tabular}

\section{State space analysis of the hierarchical flexible cell}

A state space analysis is a report of the analysis for all possible occurrences of the part in the model of the system. State space provides statistic report of the model, boundedness properties, home properties, liveness properties and fairness properties for transition in the 
model. The CPNTools software features the state space tool which is able to generate strongly connected Component Graph (SCC graph) and reachability properties for the constructed model. To generate and collect a report from the state space module we need to calculate the state space, calculate SCC graph and save the report in a notepad. The display of the nodes with specific options is available in the tool and also it can generate successors and predecessors for the nodes. In Figure 4, we present view of state space tool in CPNTools software to generate the properties for the cell.

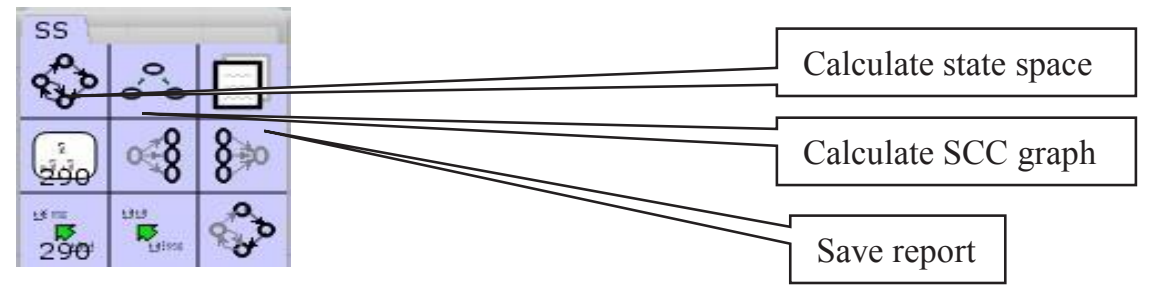

Fig. 4. State space tool in CPNTools software.

The static information of the cell is as follows: in state space, the full status found for the cell, produced 102887 nodes, 204968 arcs and the time took was around $210 \mathrm{sec}$ to generate the state space model. In state space we generate SCC graph and reachability properties. In Figure 5, we show the part of state space properties for the flexible manufacturing cell.

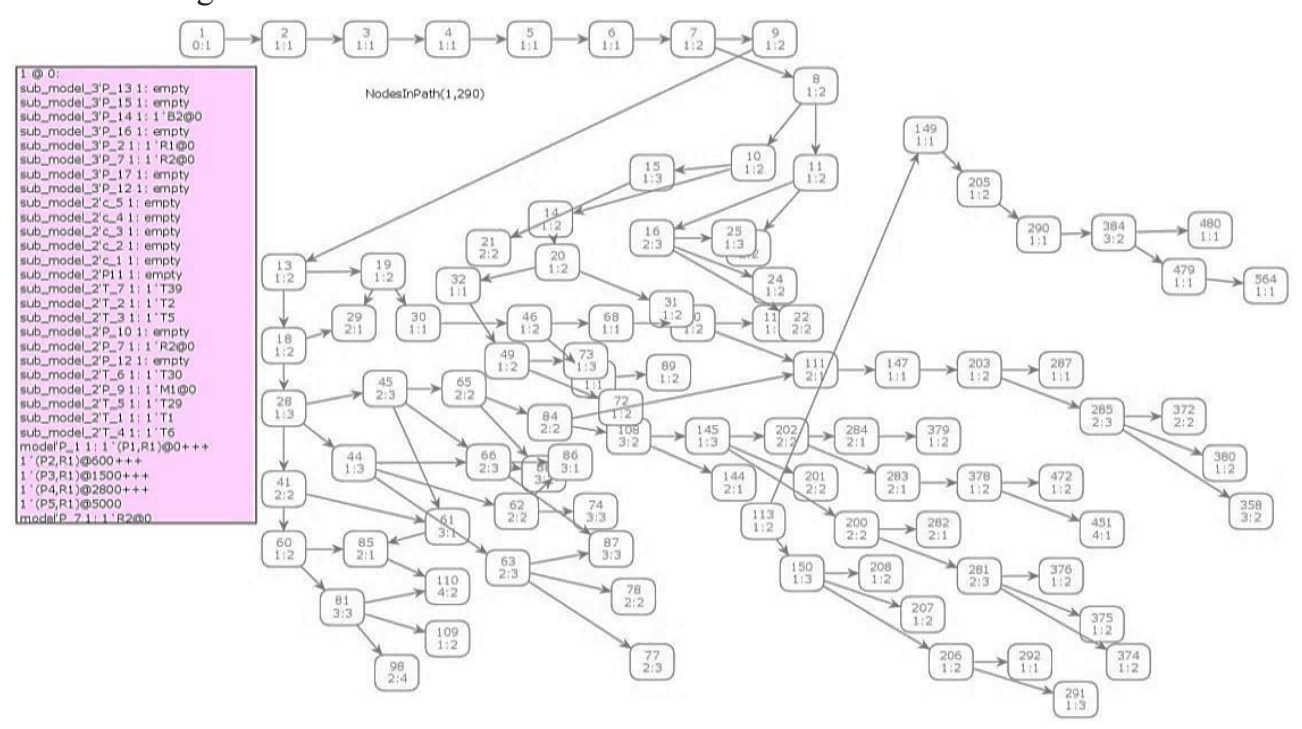

Fig. 5. State space structure view of the hierarchical model based manufacturing cell.

\subsection{Boundedness Properties in the cell}

We present the boundedness properties of the hierarchical model with the best possible multi set bounds for the cell. The best integer represents all the sections of the cell. In this section, the lower multi set bounds for each place are found empty during generating the state space. Each integer bound presents the marking situation with respect to their places. 
Respective upper multi set bounds define the marking of that place with color token value.

The best integer with best upper multi set bounds are respectively:

Table 2.Best Integer Bounds for Hierarchical Model

\begin{tabular}{|c|c|c|}
\hline $\begin{array}{l}\text { Best Integer } \\
\text { Bounds }\end{array}$ & Best Upper Multi-set Bounds & $\begin{array}{c}\text { Best Lower } \\
\text { Multi-set } \\
\text { Bounds }\end{array}$ \\
\hline model'P_10 & $\begin{array}{c}1^{`}(\mathrm{P} 1, \mathrm{R} 2)++1^{`}(\mathrm{P} 2, \mathrm{R} 2)++1^{`}(\mathrm{P} 3, \mathrm{R} 2)++1^{`}(\mathrm{P} 4, \mathrm{R} 2)++1^{`}(\mathrm{P} 5, \mathrm{R} \\
\text { 2) }\end{array}$ & Empty \\
\hline model'P_1 & $\begin{array}{c}1^{`}(\mathrm{P} 1, \mathrm{R} 1)++1^{\prime}(\mathrm{P} 2, \mathrm{R} 1)++1{ }^{\prime}(\mathrm{P} 3, \mathrm{R} 1)++1^{`}(\mathrm{P} 4, \mathrm{R} 1)++1{ }^{`}(\mathrm{P} 5, \mathrm{R} \\
\end{array}$ & Empty \\
\hline model'P_12 & $\begin{array}{c}1{ }^{\prime}(\mathrm{P} 1, \mathrm{R} 2)++1{ }^{\prime}(\mathrm{P} 2, \mathrm{R} 2)++1{ }^{\prime}(\mathrm{P} 3, \mathrm{R} 2)++1{ }^{\prime}(\mathrm{P} 4, \mathrm{R} 2)++1^{`}(\mathrm{P} 5, \mathrm{R} \\
\end{array}$ & Empty \\
\hline model'P_ 17 & 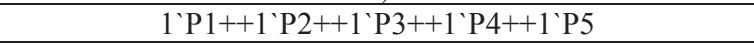 & Empty \\
\hline model'P 2 & $1 ` \mathrm{R} 1$ & Empty \\
\hline model'P 7 & $1 ` \mathrm{R} 2$ & Empty \\
\hline model'P 9 & 1`M1 & Empty \\
\hline model'T 4 & 1`T6 & Empty \\
\hline model'T 6 & $1 ` \mathrm{~T} 30$ & Empty \\
\hline model'T 7 & 1`T39 & Empty \\
\hline model'T_1 & 1`T1 & Empty \\
\hline model'T 2 & $1{ }^{\prime} \mathrm{T} 2$ & Empty \\
\hline model'T 3 & $1 ` \mathrm{~T} 5$ & Empty \\
\hline model'T_5 & $1 ` \mathrm{~T} 29$ & Empty \\
\hline sub_model_1'P_3 & $\begin{array}{c}1^{`}(\mathrm{P} 1, \mathrm{R} 1)++1^{`}(\mathrm{P} 2, \mathrm{R} 1)++1^{`}(\mathrm{P} 3, \mathrm{R} 1)++1^{\prime}(\mathrm{P} 4, \mathrm{R} 1)++1^{`}(\mathrm{P} 5, \mathrm{R} \\
1)\end{array}$ & Empty \\
\hline sub_model_1'P_4 & $11^{\prime} \mathrm{B} 1$ & Empty \\
\hline sub_model_1'P_5 & $\begin{array}{c}1^{`}(\mathrm{P} 1, \mathrm{~B} 1)++1^{\prime}(\mathrm{P} 2, \mathrm{~B} 1)++1^{\prime}(\mathrm{P} 3, \mathrm{~B} 1)++1^{\prime}(\mathrm{P} 4, \mathrm{~B} 1)++1^{`}(\mathrm{P} 5, \mathrm{~B} \\
\text { 1) }\end{array}$ & Empty \\
\hline sub_model_1'P_6 & $\begin{array}{c}1^{`}(\mathrm{P} 1, \mathrm{~B} 1)^{+++1}(\mathrm{P} 2, \mathrm{~B} 1)++1^{\prime}(\mathrm{P} 3, \mathrm{~B} 1)++1^{\prime}(\mathrm{P} 4, \mathrm{~B} 1)++1^{`}(\mathrm{P} 5, \mathrm{~B} \\
1)\end{array}$ & Empty \\
\hline sub_model_2'P11 & 1`P1++1`P2++1`P3++1`P4++1`P5 & Empty \\
\hline sub model 2'c 1 & $1 `(\mathrm{P} 1, \mathrm{~T} 5, \mathrm{M} 1)$ & Empty \\
\hline sub_model_2'c_2 & $1 `(\mathrm{P} 2, \mathrm{~T} 5, \mathrm{M} 1)$ & Empty \\
\hline sub_model_2'c_3 & 1`(P3,T30,T1,T5,T6,M1) & Empty \\
\hline sub_model_2'c_4 & $1^{`}(\mathrm{P} 4, \mathrm{~T} 30, \mathrm{~T} 39, \mathrm{M} 1)$ & Empty \\
\hline sub_model 2 'c 5 & $1 `(\mathrm{P} 5, \mathrm{~T} 30, \mathrm{~T} 29, \mathrm{~T} 2, \mathrm{M} 1)$ & Empty \\
\hline $\begin{array}{c}\text { sub_model_3'P_1 } \\
3\end{array}$ & $\begin{array}{c}1^{`}(\mathrm{P} 1, \mathrm{~B} 2)++1^{\prime}(\mathrm{P} 2, \mathrm{~B} 2)++1^{\prime}(\mathrm{P} 3, \mathrm{~B} 2)++1^{\prime}(\mathrm{P} 4, \mathrm{~B} 2)++1^{`}(\mathrm{P} 5, \mathrm{~B} \\
2)\end{array}$ & Empty \\
\hline sub_model_3'P_1 & 1 'B2 & Empty \\
\hline $\begin{array}{c}\text { sub_model_3'P_1 } \\
5\end{array}$ & $\begin{array}{c}1^{\prime}(\mathrm{P} 1, \mathrm{~B} 2)++1^{`}(\mathrm{P} 2, \mathrm{~B} 2)++1^{`}(\mathrm{P} 3, \mathrm{~B} 2)++1^{`}(\mathrm{P} 4, \mathrm{~B} 2)++1^{`}(\mathrm{P} 5, \mathrm{~B} \\
2)\end{array}$ & Empty \\
\hline $\begin{array}{c}\text { sub_model_3'P_1 } \\
6\end{array}$ & 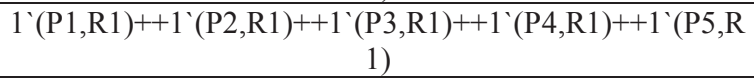 & Empty \\
\hline
\end{tabular}

The best Integer bounds for the hierarchy model describe the upper bounds and lower bounds. During generating the state space for the model the values appear in a data sheet. The observed values for integer bounds are respectively: model'P_1(upper bound=5), model'P_17 (upper bound=5), rest of integer have upper bound1 which are model'P_10, model'P_12, model'P_2, model'P_7, model'P_9, model'T_4, model'T_6, model'T_7, model'T_1, model'T_ 2 , model'T_3, model'T_5, sub_model_l1'P_3, sub_model_1'P_4, sub_model_1'P_5,sub_model_1'P_6, sub_model_2'P11,sub_model_2'c_1,sub_model_2'c_2, sub_model_2'c_3,sub_model_2'c_4,sub_model_2'c_5,sub_model_3'P_13, sub_model_3'P_1 
4,sub_model_3'P_15,sub_model_3'P_16. The lower bounds are found zero for each integer bounds.

\subsection{Home, Liveness and Fairness Properties in the cell}

The Home Properties of the cell: in this flexible cell, initial marking is not a home marking but in home marking it is possible to return to the initial marking. The marking depends on the design condition of the model. In our case the flexible cell contain no home making which is found during the generation of the state space. So, there is no way to return to the initial making in the cell.Liveness Properties of the cell: A liveness property provides information about the enabling of each transition by firing an arbitrary number of transitions in the model. In state space, dead marking means a marking of the net where no transition is enabled. This also provides information about Dead Transition Instances and Live Transition Instances in the state space. In this flexible cell we found five dead marking according to state space generated report. This model, do not contains Dead Transition Instances what means that a transition never become enabled in the cell and also found no Live Transition Instances which means a transition which always will be enabled after firing in the cell. In this, model dead marking were observed during the generation of the state space, the markings are: 290, 99497, 99496, 96100, 96069, and 96068. Fairness Properties of the cell: observing the state space we found that no infinite sequence occurred. The state space report provides the order of finite transitions in the cell.

\section{Conclusions}

In this paper we described the modeling techniques and state space structure of the flexible manufacturing cell using CPNTools software implementing the hierarchical formalism. The contribution of this work is modeling and simulation of flexible cell and identifying the behavior of the hierarchical model. The model carry significant characteristic to identify the status of the places and transitions. Also the state space analysis provides static reports for the model with features which includes the liveness properties, boundedness properties, fairness properties to analyze the simulation process.

The paper published has been sponsored under the Erasmus mundus partnership program agreement vide number 2014-0855/001-001 coordinated by and between University of Oradea and City University of London Under Action Plan 2 for the year 2015-2018.

\section{References}

1. C. Soren, L. M. Kristensen, Lscs 01: Petri Net Approaches for Modelling and Validation (2003)

2. K. Jensen, Proceedings of $2^{\text {nd }}$ International Colloquium on Petri Net Technologies for Modelling Communication Based Systems, Berlin (2001)

3. K. Jensen, Formal Methods in System Design, Kluwer Academic Publishers 9 (1-2) 7 (1996)

4. N. Viswanadham, Y. Narahari, Proc. IEEE Int. Conf. Robot. Automat 4 (1987).

5. K. Jensen, Colored Petri Nets: Basic Concepts, Analysis Methods and Practical Use 1, Springer - Verlag, (1992)

6. http://cpntools.org/ accessed on (2015) 